\title{
Pentaquark Magnetic Moments In Different Models
}

\author{
Y.-R. Liu, P.-Z. Huang, W.-Z. Deng, and X.-L. Chen \\ Department of Physics, Peking University, BEIJING 100871, CHINA \\ Shi-Lin Zhu* \\ Department of Physics, Peking University, BEIJING 100871, CHINA \\ The Key Laboratory of Heavy Ion Physics, Ministry of Education, Beijing 100871, China
}

(Dated: August 19, 2018)

\begin{abstract}
We calculate the magnetic moments of the pentaquark states from different models and compare our results with predictions of other groups.

PACS numbers: 12.39.Mk, 14.20.-C, 12.39.-x

Keywords: Pentaquark, Magnetic Moments
\end{abstract}

\section{INTRODUCTION}

LEPS Collaboration first discovered a very narrow baryon state around $1540 \mathrm{MeV}$ with strangeness $S=+1$, which was confirmed by DIANA [2], CLAS [3, 7], SAPHIR [4], ITEP [5] Collaboration. The positive strangeness means that the valence quark number inside this baryon is five. Such a state is definitely beyond the conventional quark model. Now it is called the $\Theta^{+}$pentaquark. Later, NA49 Collaboration found evidence for the existence of another narrow baryon resonance with $Q=-2, S=-2, I=3 / 2$ and mass of $(1.862 \pm 0.002) \mathrm{GeV} / \mathrm{c}^{2}$ from high energy collision experiment [6]. Experimentally there is evidence that $\Theta^{+}$particle may be an isoscalar from the featureless $p K^{+}$ spectrum 3, 4, 7]. The parity and angular moment of the $\Theta^{+}$particle have not been determined experimentally.

One should keep in mind that Quantum Chromodynamics (QCD) as the underlying theory of the strong interaction does allow the possible existence of the non-conventional hadrons with the quark content other than $q \bar{q}$ or $q q q$. Some examples are glueballs $(g g, g g g, \cdots)$, hybrid mesons $(q \bar{q} g)$, and other multi-quark states $(q q \bar{q} \bar{q}, q q q q \bar{q}, q q q \bar{q} \bar{q} \bar{q}$, $q q q q q q, \cdots)$. In fact, hybrid mesons are found to mix freely with conventional mesons in the large $N_{c}$ limit $[8]$. However, despite extensive experimental searches in the past two decades, none of these states has been firmly established until the surprising discovery of pentaquarks $[9]$.

These experiments have triggered heated discussions of the interpretation of these resonances and their quantum numbers [11]- 35]. The partial motivation of the recent experimental search of the pentaquark state came from the work by Diakonov et al. [10]. They proposed the existence of the $J^{P}=\frac{1}{2}^{+} \Theta^{+}$pentaquark at $1530 \mathrm{MeV}$ with a very narrow width in the chiral soliton model (CSM).

However, such a picture is challenged by Refs. 11, 12, 13]. It was pointed out in 11] that identifying $N(1710)$ as a member of the anti-decuplet in the CSM is kind of arbitrary. Using a more reasonable value for the $N(1710)$ decay width as the input may lead to a big width of $\Theta^{+}$particle exceeding the present experimental upper bound. Another serious challenge to the chiral soliton model came from the large $N_{c}$ consistency consideration by Cohen [12], in which he concluded that the prediction for $\Theta^{+}$properties based on collective quantization of CSM was not valid. The reason is that collective quantization is legitimate only for excitations which vanish as $N_{c} \rightarrow \infty$. In Ref. 13] it was shown that the exotic state may be an artifact of the rigid rotator approach to the Skyrme model for large $N_{c}$ and small $m_{K}$. Moreover, the mass of the pentaquark state with the quark content $(d s d s \bar{u})$ is rigourously predicted in the chiral soliton model to be $2070 \mathrm{MeV}$, which is $210 \mathrm{MeV}$ higher than the experimental value measured by NA49 Collaboration 6 .

Although it is very difficult to obtain a low-mass $\Theta^{+}$pentaquark using the picture of constituent quarks, it is still possible to accommodate such a low mass in other theoretical approaches. For example, the $\Theta^{+}$pentaquark mass from QCD sum rule formalism [14, 15] and lattice QCD simulation [16] is consistent with the experimental value. Treating the pentaquark as the bound state of two tight bound diquarks and one anti-quark or as the bound state of a diquark and a triquark may also lower its mass [17, 18, 19].

Because of its low mass, most of theoretical work postulated its angular momentum to be one half. But it was pointed out that the possibility of $J=\frac{3}{2}$ still can not be excluded completely [20]. The parity of the $\Theta^{+}$pentaquark is

*Electronic address: zhusl@th.phy.pku.edu.cn 
still a big puzzle. Up to now theoretical papers in the literature can be roughly classified into two types according to their assumption of $\Theta^{+}$parity. It's interesting to note that QCD sum rule approach [14, 15], lattice QCD calculation [16] and some models [21, 22, 23] favor the negative parity. Many other theoretical papers assume positive parity for $\Theta^{+}$pentaquark as in the original Ref. 10]. Recently some typical examples are the clustered quark models proposed by Jaffe and Wilczek [17], Shuryak and Zahed [19], and by Karliner and Lipkin [18].

The $\Theta^{+}$parity is very challenging. But the more challenging one to theorists is the extreme narrow width of the $\Theta^{+}$particle. This baryon has such a narrow width that all experiments can only set an upper bound. There is even stringent limit arising from recent analysis that its width is less than several MeV [24, 25, 26, 27].

Experience with conventional excited hadrons shows that their widths are around one hundred MeV or even bigger if they lie $100 \mathrm{MeV}$ above threshold and decay through $\mathrm{S}$-wave or $\mathrm{P}$-wave. For comparison, the $S=-1$ hyperon $\Lambda(1520) D_{03}$ state is in the same mass region as the $\Theta^{+}$pentaquark. Its angular momentum and parity is $J^{P}=\frac{3}{2}^{-}$. Its dominant two-body decay is of D-wave with final states $N \bar{K}, \Sigma \pi$. With a smaller phase space and higher partial wave, the width of $\Lambda(1520)$ is $15.6 \pm 1.0 \mathrm{MeV}$ [9]. In contrast, $\Theta^{+}$decays through either $\mathrm{S}$-wave or $\mathrm{P}$-wave with a total width less than several $\mathrm{MeV}$, corresponding to negative or positive parity respectively. Such a comparison is really disturbing.

If these states are further established and confirmed to have such a narrow width, there must be a mysterious selection rule which is absent from the conventional hadron interaction. Uncovering and understanding such an underlying selection rule would be a top priority to theorists.

We have estimated the mass of the pentaquark state with QCD sum rules and found that the lowest pentaquark state lies around $(1.55 \pm 0.15) \mathrm{GeV}$ [14]. The baryon magnetic moment is another fundamental observable as its mass, which encodes information of the underlying quark structure and dynamics. We have also employed the light cone QCD sum rules to extract the magnetic moment of the $\Theta^{+}$particle and found its value is quite small [28].

A first-principle dynamical determination of the pentaquark quantum numbers is still not feasible right now. In this paper, we shall start from different models available in literature and study their phenomenological prediction, namely the magnetic moments of pentaquarks. We shall calculate the pentaquark magnetic moments in several typical models. They include (1) Jaffe and Wilczek's model [17], (2) Shuryak and Zahed's model [19], (3) Karliner and Lipkin's model [18], (4) Strottman's model [29]. Different models generally yield different values. This in turn will affect the photo-production and electro-production cross sections of pentaquarks. Such a study will deepen our knowledge of pentaquark states and may help us explore its dynamics and distinguish so many models in the literature.

Our paper is organized as follows: Section I is an introduction. A brief review of this field is presented. In Section II-III we calculate pentaquark magnetic moments in the Jaffe and Wilczek's model and Shuryak and Zahed's model respectively. In Section IV we perform the same calculation in Lipkin and Karliner's model treating pentaquarks as a bound state of a diquark and triquark. In Section V we perform the calculation in Strottman's model, treating pentaquarks as a cluster of four quarks and one anti-quark. Our discussions are given in Section VI, where we also compare our result with other groups' prediction.

\section{PENTAQUARK AS A BOUND STATE OF TWO SCALAR DIQUARKS AND ONE ANTI-QUARK}

Jaffe and Wilczek (JW) suggested that the observed $\Theta^{+}$state is composed of an anti-strange quark and two highly correlated up and down quark pairs arising from strong color-spin correlation [17]. They treated the diquarks as point-like particles obeying Bose statistics and predicted the isospin $3 / 2$ multiplet of $\Xi_{5}^{--}(d d s s \bar{u})$ with $S=-2$ and $J^{P}=\frac{1}{2}^{+}$around $1750 \mathrm{MeV}$. Such a state with the same quantum number was observed by NA49 but with a much higher mass at $1860 \mathrm{MeV}$ [6].

According to JW's model, there will be a $J=\frac{1}{2} S U_{f}(3)$ antidecuplet and an octet. But some pentaquarks in the octet may mix with those in the antideculplet. Three pentaquarks have "pure" components: $\Theta^{+}(u d u d \bar{s}), \Xi_{5}^{+}(u s u s \bar{d})$ and $\Xi_{5}^{--}(d s d s \bar{u}) . \Theta^{+}$is at the apex of the antidecuplet and the other two are at the bottom corners. They also predicted their corresponding masses of these exotic baryons. In this section, we will calculate the magnetic moments of these pentaquarks in this model.

The diquark arises from the strong chromomagnetic attraction or instanton interaction between up quark and down quark when they are in the color $\overline{\mathbf{3}}$ channel. In JW's model, each diquark is in the antisymmetric $\overline{\mathbf{3}}_{\mathbf{c}}$ color representation. The spin wave function of a scalar diquark is antisymmetric and the space wave function is symmetric. Thus the scalar diquark has positive parity. Because of the Pauli principle, the total wave function of the diquark

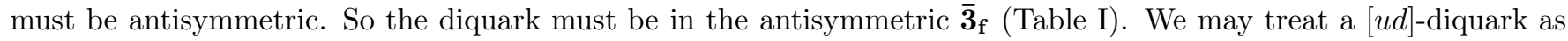
an "effective" $s$-antiquark, $[d s]$-diquark as $u$-antiquark, and $[s u]$-diquark as $d$-antiquark in flavor and color symmetry $S U(3)_{f} \otimes S U(3)_{c}$.

The total wave function of the two diquark system must be symmetric under the diquark exchange. The color part 


\begin{tabular}{ccccc}
\hline$\left(Y_{1}, I_{1}\right)$ & $\left(Y_{2}, I_{2}\right)$ & $(Y, I)$ & $I_{3}$ & Flavor Wave Functions \\
\hline$\left(\frac{1}{3}, \frac{1}{2}\right)$ & $\left(\frac{1}{3}, \frac{1}{2}\right)$ & $\left(\frac{2}{3}, 0\right)$ & 0 & {$[u d]=\frac{1}{\sqrt{2}}(u d-d u)$} \\
$\left(\frac{1}{3}, \frac{1}{2}\right)$ & $\left(-\frac{2}{3}, 0\right)$ & $\left(-\frac{1}{3}, \frac{1}{2}\right)$ & $\frac{1}{2}$ & {$[u s]=\frac{1}{\sqrt{2}}(u s-s u)$} \\
$\left(-\frac{2}{3}, 0\right)$ & $\left(\frac{1}{3}, \frac{1}{2}\right)$ & $\left(-\frac{1}{3}, \frac{1}{2}\right)$ & $-\frac{1}{2}$ & {$[d s]=\frac{1}{\sqrt{2}}(d s-s d)$} \\
\hline
\end{tabular}

TABLE I: Diquark flavor wave functions. $Y, I$ and $I_{3}$ are hypercharge, isospin and the third component of isospin respectively. The subscripts 1 and 2 represent two quarks inside the diquark.

is antisymmetric. The spin wave function is certainly symmetric in the case of scalar diquarks. Since the $\Theta^{+}$particle is assumed to be an isoscalar, the diquark-diquark system in $\Theta^{+}$also has $I=0$. Its $S U_{f}(3)$ flavor wave function is symmetric $\overline{\mathbf{6}}_{\mathbf{f}}$. So the spatial wave function of diquark-diquark is antisymmetric, which implies the orbital angular momentum between the two diquarks is odd. Thus the parity of the two diquark system is negative. Finally the two diquarks combine with an antiquark to form the exotic pentaquark state. The total angular momentum and parity of the pentaquark will be $J^{P}=\frac{1}{2}^{+}$or $\frac{3}{2}^{+}$. In general, lower angular momentum implies lower mass. So $J^{P}=\frac{1}{2}^{+}$is adpoted in Ref. [17]. Pentaquarks belong to either the flavor octet $\mathbf{8}_{\mathbf{f}}$ or an anti-decuplet $\overline{\mathbf{1 0}}_{\mathbf{f}}$ in JW's model. The resulting flavor wave functions are listed in Table

\begin{tabular}{cc||cc}
\hline$\left(Y, I, I_{3}\right)$ & $\mathbf{1 0}$ & $\left(Y, I, I_{3}\right)$ & $\mathbf{8}$ \\
\hline$(2,0,0)$ & {$[u d]^{2} \bar{s}$} & - & - \\
$\left(1, \frac{1}{2}, \frac{1}{2}\right)$ & $\sqrt{\frac{2}{3}}[u d][u s]_{+} \bar{s}+\sqrt{\frac{1}{3}}[u d]^{2} \bar{d}$ & $\left(1, \frac{1}{2}, \frac{1}{2}\right)$ & $\sqrt{\frac{1}{3}}[u d][u s]_{+} \bar{s}-\sqrt{\frac{2}{3}}[u d]^{2} \bar{d}$ \\
$\left(1, \frac{1}{2},-\frac{1}{2}\right)$ & $\sqrt{\frac{2}{3}}[u d][d s]_{+} \bar{s}+\sqrt{\frac{1}{3}}[u d]^{2} \bar{u}$ & $\left(1, \frac{1}{2}, \frac{1}{2}\right)$ & $\sqrt{\frac{1}{3}}[u d][d s]_{+} \bar{s}-\sqrt{\frac{2}{3}}[u d]^{2} \bar{u}$ \\
$(0,1,1)$ & $\sqrt{\frac{2}{3}}[u d][u s]_{+} \bar{d}+\sqrt{\frac{1}{3}}[u s]^{2} \bar{s}$ & $(0,1,1)$ & $\sqrt{\frac{1}{3}}[u d][u s]_{+} \bar{d}-\sqrt{\frac{2}{3}}[u s]^{2} \bar{s}$ \\
$(0,1,0)$ & $\sqrt{\frac{1}{3}}\left([u d][d s]_{+} \bar{d}+[u d][u s]_{+} \bar{u}\right.$ & $(0,1,0)$ & $\sqrt{\frac{1}{6}}\left([u d][d s]_{+} \bar{d}+[u d][u s]_{+} \bar{u}\right)$ \\
& $\left.+[u s][d s]_{+} \bar{s}\right)$ & & $-\sqrt{\frac{2}{3}}[u s][d s]_{+} \bar{s}$ \\
$(0,1,-1)$ & $\sqrt{\frac{2}{3}}[u d][d s]_{+} \bar{u}+\sqrt{\frac{1}{3}}[d s]^{2} \bar{s}$ & $(0,1,-1)$ & $\sqrt{\frac{1}{3}}[u d][d s]_{+} \bar{u}-\sqrt{\frac{2}{3}}[d s]^{2} \bar{s}$ \\
$\left(-1, \frac{3}{2}, \frac{3}{2}\right)$ & {$[u s]^{2} \bar{d}$} & - & - \\
$\left(-1, \frac{3}{2}, \frac{1}{2}\right)$ & $\sqrt{\frac{2}{3}}[u s][d s]_{+} \bar{d}+\sqrt{\frac{1}{3}}[u s]^{2} \bar{u}$ & $\left(-1, \frac{1}{2}, \frac{1}{2}\right)$ & $\sqrt{\frac{1}{3}}[u s][d s]_{+} \bar{d}-\sqrt{\frac{2}{3}}[u s]^{2} \bar{u}$ \\
$\left(-1, \frac{3}{2},-\frac{1}{2}\right.$ & $\sqrt{\frac{2}{3}}[d s][u s]_{+} \bar{u}+\sqrt{\frac{1}{3}}[d s]^{2} \bar{d}$ & $\left(-1, \frac{1}{2},-\frac{1}{2}\right)$ & $\sqrt{\frac{1}{3}}[d s][u s]_{+} \bar{u}-\sqrt{\frac{2}{3}}[d s]^{2} \bar{d}$ \\
$\left(-1, \frac{3}{2},-\frac{3}{2}\right)$ & {$[d s]^{2} \bar{u}$} & - & - \\
- & - & $(0,0,0)$ & $\sqrt{\frac{1}{2}}\left([u d][d s]_{+} \bar{d}-[u d][u s]_{+} \bar{u}\right)$ \\
\hline
\end{tabular}

TABLE II: Flavor wave functions in Jaffe and Wilczek's model $[17] .\left[q_{1} q_{2}\right]\left[q_{3} q_{4}\right]_{+}=\sqrt{\frac{1}{2}}\left(\left[q_{1} q_{2}\right]\left[q_{3} q_{4}\right]+\left[q_{3} q_{4}\right]\left[q_{1} q_{2}\right]\right)$ or $\left[q_{1} q_{2}\right]^{2}=$ $\left[q_{1} q_{2}\right]\left[q_{1} q_{2}\right]$ is the diquark-diquark part.

The magnetic moment of a compound system is the sum of the magnetic moments of its constituents including spin and orbital contributions,

$$
\vec{\mu}=\sum_{i} \overrightarrow{\mu_{i}}=\sum_{i}\left(g_{i} \overrightarrow{s_{i}}+\overrightarrow{l_{i}}\right) \mu_{i}
$$

where $g_{i}$ is the $g$-factor of $i$-th constituent and $\mu_{i}$ is the magneton of the $i$-th constituent

$$
\mu_{i}=\frac{e_{i}}{2 m_{i}}
$$

Treating the scalar diquarks as composite bosons, the pentaquark can be regarded as a three-body system consisting of two scalar bosons and a spin- $\frac{1}{2}$ fermion. Thus

$$
\begin{aligned}
\vec{\mu} & =\left(g_{1} \overrightarrow{0}+\overrightarrow{l_{1}}\right) \mu_{1}+\left(g_{2} \overrightarrow{0}+\overrightarrow{l_{2}}\right) \mu_{2}+\left(g_{3} \frac{\overrightarrow{1}}{2}+\overrightarrow{0}\right) \mu_{3}, \\
& =\overrightarrow{l_{1}} \mu_{1}+\overrightarrow{l_{2}} \mu_{2}+g_{3} \frac{\overrightarrow{1}}{2} \mu_{3} .
\end{aligned}
$$


It is clear from the above equation that the pentaquark magnetic moment arises from the spin of the antiquark and the relative angular momentum between the two scalar diquarks which are labelled as 1 and 2 .

The orbital magnetic moment $\mu_{l}$ of a pentaquark in this model is defined as

$$
\mu_{l} \vec{l}=\overrightarrow{l_{1}} \mu_{1}+\overrightarrow{l_{2}} \mu_{2}
$$

The spatial part of the diquark-diquark wave function can be separated into the overall center-of-mass motion and the relative motion

$$
\Psi_{\text {spatial }}=\Psi(\mathbf{R}) \psi(\mathbf{r})
$$

where

$$
\mathbf{R}=\frac{m_{1} \mathbf{r}_{1}+m_{2} \mathbf{r}_{2}}{m_{1}+m_{2}}
$$

is the position of the center of mass and

$$
\mathbf{r}=\mathbf{r}_{1}-\mathbf{r}_{2}
$$

is the relative position between the two diquarks. Now we calculate each diquark's orbital angular momentum.

$$
\mathbf{l}_{1}=\mathbf{r}_{1} \times \mathbf{p}_{1}=\left(\mathbf{R}+\frac{m_{2}}{m_{1}+m_{2}} \mathbf{r}\right) \times\left(\frac{m_{1}}{m_{1}+m_{2}} \mathbf{P}+\mathbf{p}\right)
$$

Since the pentaquark is a bound states of multi-quarks, the average momentum of every quark is zero: $\left\langle\mathbf{p}_{\mathbf{i}}\right\rangle=0$. So we have $\langle\mathbf{P}\rangle=0$ and $\langle\mathbf{p}\rangle=0$, from which we get:

$$
\begin{aligned}
\left\langle\mathbf{l}_{1}\right\rangle & =\frac{m_{2}}{m_{1}+m_{2}}\langle\mathbf{r} \times \mathbf{p}\rangle+\frac{m_{1}}{m_{1}+m_{2}}\langle\mathbf{R} \times \mathbf{P}\rangle \\
& =\frac{m_{2}}{m_{1}+m_{2}}\langle\mathbf{l}\rangle+\frac{m_{1}}{m_{1}+m_{2}}\langle\mathbf{L}\rangle .
\end{aligned}
$$

$\mathbf{l}=\mathbf{r} \times \mathbf{p}$ is just the diquark-diquark's relative orbital angular momentum. $\mathbf{L}=\mathbf{R} \times \mathbf{P}$ is the diquark-diquark's orbital angular momentum for the motion of center of mass. The pentaquark states are assumed to have only a relative angular momentum $l=1$ between the diquarks. Thus $\langle\mathbf{L}\rangle=0$, which is manifest in the center of mass frame. Therefore, we get

$$
\begin{aligned}
& \left\langle\mathbf{l}_{1}\right\rangle=\frac{m_{2}}{m_{1}+m_{2}}\langle\mathbf{l}\rangle . \\
& \left\langle\mathbf{l}_{2}\right\rangle=\frac{m_{1}}{m_{1}+m_{2}}\langle\mathbf{l}\rangle .
\end{aligned}
$$

Now for the magnetic moment of the two diquark system, we have

$$
\begin{aligned}
\mu_{l} \vec{l} & =\frac{m_{2} \mu_{1}}{m_{1}+m_{2}} \vec{l}+\frac{m_{1} \mu_{2}}{m_{1}+m_{2}} \vec{l}, \\
\mu_{l} & =\frac{m_{2} \mu_{1}}{m_{1}+m_{2}}+\frac{m_{1} \mu_{2}}{m_{1}+m_{2}} .
\end{aligned}
$$

Finally the magnetic moment of a pentaquark reads

$$
\begin{aligned}
\mu & =\left\langle 2 \mu_{\bar{q}} \frac{\overrightarrow{1}}{2}+\mu_{l} \vec{l}\right\rangle\left(J_{z}=\frac{1}{2}\right) \\
& =\left(\left\langle 10 \frac{1}{2} \frac{1}{2} \mid \frac{1}{2} \frac{1}{2}\right\rangle^{2}-\left\langle 11 \frac{1}{2}-\frac{1}{2} \mid \frac{1}{2} \frac{1}{2}\right\rangle^{2}\right) \mu_{\bar{q}}+\left\langle 11 \frac{1}{2}-\frac{1}{2} \mid \frac{1}{2} \frac{1}{2}\right\rangle^{2} \mu_{l}
\end{aligned}
$$

We present the expressions of the pentaquark magnetic moments in Table III and IV We use $m_{u}=m_{d}=360$ $\mathrm{MeV}, m_{s}=500 \mathrm{MeV}$ and two sets of diquark mass parameters to compute the magnetic moments of these $J^{P}=\frac{1}{2}^{+}$ pentaquarks. The numerical results are summarized in Table $\nabla$ 


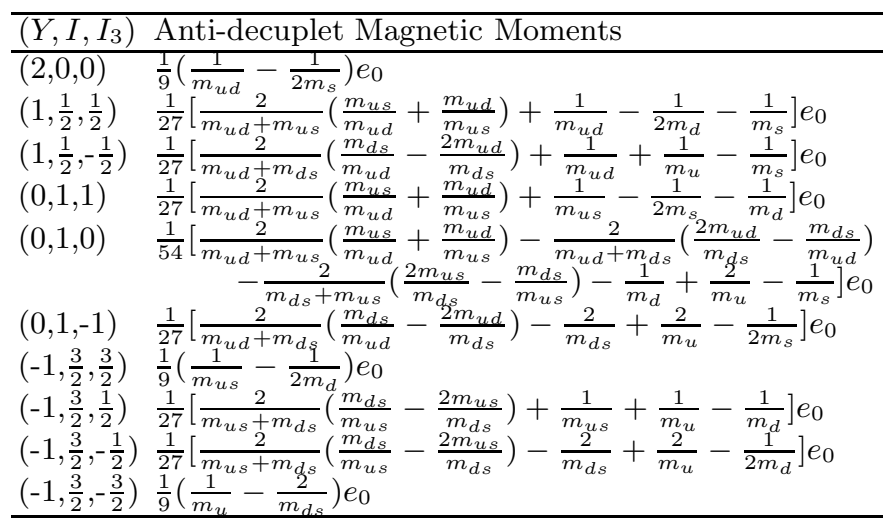

TABLE III: Expressions of the magnetic moments of the anti-decuplet pentaquarks in Jaffe and Wilczek's model [17], where $e_{0}$ is the charge unit.

\begin{tabular}{ll}
\hline$\left(Y, I, I_{3}\right)$ & Octet Pentaquark Magnetic Moments \\
\hline$\left(1, \frac{1}{2}, \frac{1}{2}\right)$ & $\frac{1}{27}\left[\frac{1}{m_{u d}+m_{u s}}\left(\frac{m_{u s}}{m_{u d}}+\frac{m_{u d}}{m_{u s}}\right)+\frac{2}{m_{u d}}-\frac{1}{m_{d}}-\frac{1}{2 m_{s}}\right] e_{0}$ \\
$\left(1, \frac{1}{2},-\frac{1}{2}\right)$ & $\frac{1}{27}\left[\frac{1}{m_{u d}+m_{d s}}\left(\frac{m_{d s}}{m_{u d}}-\frac{2 m_{u d}}{m_{d s}}\right)+\frac{2}{m_{u d}}+\frac{2}{m_{u}}-\frac{1}{2 m_{s}}\right] e_{0}$ \\
$(0,1,1)$ & $\frac{1}{27}\left[\frac{1}{m_{u d}+m_{u s}}\left(\frac{m_{u s}}{m_{u d}}+\frac{m_{u d}}{m_{u s}}\right)+\frac{2}{m_{u s}}-\frac{1}{m_{s}}-\frac{1}{2 m_{d}}\right] e_{0}$ \\
$(0,1,0)$ & $\frac{1}{54}\left[\frac{1}{m_{u d}+m_{u s}}\left(\frac{m_{u s}}{m_{u d}}+\frac{m_{u d}}{m_{u s}}\right)+\frac{1}{m_{u d}+m_{d s}}\left(\frac{m_{d s}}{m_{u d}}-\frac{2 m_{u d}}{m_{d s}}\right)\right.$ \\
& $\left.\quad+\frac{4}{m_{d s}+m_{u s}}\left(\frac{m_{d s}}{m_{u s}}-\frac{2 m_{u s}}{m_{d s}}\right)+\frac{1}{m_{u}}-\frac{1}{2 m_{d}}-\frac{2}{m_{s}}\right] e_{0}$ \\
$(0,1,-1)$ & $\frac{1}{27}\left[\frac{1}{m_{u d}+m_{d s}}\left(\frac{m_{d s}}{m_{u d}}-\frac{2 m_{u d}}{m_{d s}}\right)-\frac{4}{m_{d s}}+\frac{1}{m_{u}}-\frac{1}{m_{s}}\right] e_{0}$ \\
$\left(-1, \frac{1}{2}, \frac{1}{2}\right)$ & $\frac{1}{27}\left[\frac{1}{m_{u s}+m_{d s}}\left(\frac{m_{d s}}{m_{u s}}-\frac{2 m_{u s}}{m_{d s}}\right)+\frac{2}{m_{u s}}+\frac{2}{m_{u}}-\frac{1}{2 m_{d}}\right] e_{0}$ \\
$\left(-1, \frac{1}{2},-\frac{1}{2}\right)$ & $\frac{1}{27}\left[\frac{1}{m_{u s}+m_{d s}}\left(\frac{m_{d s}}{m_{u s}}-\frac{2 m_{u s}}{m_{d s}}\right)-\frac{4}{m_{d s}}+\frac{1}{m_{u}}-\frac{1}{m_{d}}\right] e_{0}$ \\
$(0,0,0)$ & $\frac{1}{18}\left[\frac{1}{m_{u d}+m_{u s}}\left(\frac{m_{u s}}{m_{u d}}+\frac{m_{u d}}{m_{u s}}\right)+\frac{1}{m_{u d}+m_{d s}}\left(\frac{m_{d s}}{m_{u d}}-\frac{2 m_{u d}}{m_{d s}}\right)+\frac{1}{m_{u}}-\frac{1}{2 m_{d}}\right] e_{0}$ \\
\hline
\end{tabular}

TABLE IV: Expressions of the magnetic moments of the octet pentaquarks in Jaffe and Wilczek's model [17], where $e_{0}$ is the charge unit.

For $\Theta^{+}(1540)$, we have $\mu_{\Theta^{+}}=0.081 \frac{e_{0}}{2 m_{N}} \simeq 0.13 \frac{e_{0}}{2 M_{\Theta^{+}}}$with Set I parameters from Ref. [18], while $\mu_{\Theta^{+}} \simeq 0.47 \frac{e_{0}}{2 M_{\Theta^{+}}}$ with Set II parameters from Ref. [17]. $e_{0}$ is the positive charge unit. For the newly observed pentaquark $\Xi_{5}^{--}(1860)$ which lies at $\left(-1,-\frac{3}{2}\right)$ of $I_{3}-Y$ diagram and is composed of $d d s s \bar{u}$, we have $\mu_{\Xi_{5}^{--}}=0.116 \frac{e_{0}}{2 m_{N}} \simeq 0.23 \frac{e_{0}}{2 M_{\Xi_{5}^{-}}}$with set I masses, while $\mu_{\Xi_{5}^{--}} \simeq-0.23 \frac{e_{0}}{2 M_{\Xi_{5}^{--}}}$with set II masses.

The magnetic moments are quite sensitive to the diquark mass parameters. In our case, the pentaquark wave functions are constructed using symmetries only. The physical particle may be the mixture of those states at the same position in $I_{3}-Y$ diagram. For simplicity we have ignored all possible mixing effects. Experimentally, it will be very challenging to identify those pentaquarks without exotic quantum numbers.

\section{PENTAQUARK AS A BOUND STATE OF ONE SCALAR DIQUARK, ONE TENSOR DIQUARK AND ONE ANTI-QUARK}

Replacing the pair of scalar diquarks with one scalar and one tensor diquark, Shuryak and Zahed argued that the pentaquark mass may be lowered in this way [19]. The reason is that the orbital excitation between diquarks is no longer necessary, although the tensor diquark mass is slightly higher than the scalar one. The orbital excitation between the up and down quarks inside the tensor diquark ensures that the overall parity of the $\Theta^{+}$particle is positive. They claimed that they can get much lighter states than in JW's model.

Now the tensor diquark is in the antisymmetric $\overline{\mathbf{3}}_{\mathbf{c}}$ representation and its flavor is $\overline{\mathbf{3}}_{\mathbf{f}}$. To get an $I=0 \Theta^{+}$, the diquark-diquark must be still in the symmetric flavor $\overline{\mathbf{6}}_{\mathbf{f}}$ representation. Thus the flavor wave functions of the pentaquark states remain the same as in JW's model after adding the single anti-quark. However, the total angular momentum of the tensor diquark is chosen to be $J=1$. The Pauli principle demands that there is at least an orbital excitation $l=1$ between two quarks inside the tensor diquark. Thus the total spin of the two quarks inside the tensor 


\begin{tabular}{c|c|c||c|c|c}
\hline State & \multicolumn{2}{|c||}{$\mathbf{1 0}$} & State & \multicolumn{2}{c}{$\mathbf{8}$} \\
\hline$\left(Y, I, I_{3}\right)$ & set I & set II & $\left(Y, I, I_{3}\right)$ & set I & set II \\
\hline$(2,0,0)$ & 0.08 & 0.29 & - & - & - \\
$\left(1, \frac{1}{2}, \frac{1}{2}\right)$ & 0.037 & 0.22 & $\left(1, \frac{1}{2}, \frac{1}{2}\right)$ & 0.018 & 0.21 \\
$\left(1, \frac{1}{2},-\frac{1}{2}\right)$ & 0.12 & 0.22 & $\left(1, \frac{1}{2},-\frac{1}{2}\right)$ & 0.50 & 0.65 \\
$(0,1,1)$ & -0.009 & 0.14 & $(0,1,1)$ & 0.007 & 0.14 \\
$(0,1,0)$ & 0.06 & 0.12 & $(0,1,0)$ & -0.13 & -0.13 \\
$(0,1,-1)$ & 0.13 & 0.09 & $(0,1,-1)$ & -0.27 & -0.41 \\
$\left(-1, \frac{3}{2}, \frac{3}{2}\right)$ & -0.06 & 0.06 & - & - & - \\
$\left(-1, \frac{3}{2}, \frac{1}{2}\right)$ & 0 & 0 & $\left(-1, \frac{1}{2}, \frac{1}{2}\right)$ & 0.41 & 0.46 \\
$\left(-1, \frac{1}{2},-\frac{1}{2}\right)$ & 0.06 & -0.06 & $\left(-1, \frac{1}{2},-\frac{1}{2}\right)$ & -0.35 & -0.52 \\
$\left(-1, \frac{3}{2},-\frac{3}{2}\right)$ & 0.12 & -0.12 & - & - & - \\
- & - & - & $(0,0,0)$ & 0.25 & 0.37 \\
\hline
\end{tabular}

TABLE V: Numerical results of the magnetic moments of $\overline{\mathbf{1 0}}$ and $\mathbf{8}$ pentaquarks in unit of $\mu_{N}$ in Jaffe and Wilczek's model [17. For set I we use $m_{u d}=720 \mathrm{MeV}, m_{u s}=m_{d s}=900 \mathrm{MeV}$ from Ref. [18] and for set II we use $m_{u d}=420 \mathrm{MeV}$, $m_{u s}=m_{d s}=600 \mathrm{MeV}$ from Ref. [17].

diquark is one. So the parity of the tensor quark is negative. As a result, all of the eighteen pentaquark states have positive parity as in JW's model while the spin of the pentaquark is either $J=\frac{1}{2}$ or $\frac{3}{2}$.

The magnetic moment of a pentaquark in SZ's model is

$$
\begin{aligned}
\vec{\mu} & =\left(g_{1} \overrightarrow{0}+\overrightarrow{0}\right) \mu_{1}+\left(g_{2} \overrightarrow{1}+\overrightarrow{0}\right) \mu_{2}+\left(g_{3} \frac{\overrightarrow{1}}{2}+\overrightarrow{0}\right) \mu_{3}, \\
& =g_{2} \overrightarrow{1} \mu_{2}+g_{3} \frac{\overrightarrow{1}}{2} \mu_{3} .
\end{aligned}
$$

The magnetic moment of a pentaquark comes from the spin of the antiquark and the spin of the tensor diquark which is labelled 2.

The tensor diquark's $g$-factor can be calculated from its two components of an $i$ - quark and a $j$ - quark

$$
g_{2} \overrightarrow{1} \mu_{2}=\left(g \frac{\overrightarrow{1}}{2}+\overrightarrow{l_{i}}\right) \mu_{i}+\left(g \frac{\overrightarrow{1}}{2}+\overrightarrow{l_{j}}\right) \mu_{j},
$$

where $g=2$ is the quark $g$ factor. The orbital magnetic moment $\mu_{l}$ of the tensor diquark in this model is

$$
\begin{gathered}
\mu_{l} \vec{l}=\overrightarrow{l_{i}} \mu_{i}+\overrightarrow{l_{j}} \mu_{j}, \\
\mu_{l}=\frac{m_{j} \mu_{i}}{m_{i}+m_{j}}+\frac{m_{i} \mu_{j}}{m_{i}+m_{j}} .
\end{gathered}
$$

The $g$ factor of the tensor diquark can be evaluated with

$$
\begin{aligned}
& g_{2} \overrightarrow{1} \mu_{2}=2 \frac{\overrightarrow{1}}{2} \mu_{i}+2 \frac{\overrightarrow{1}}{2} \mu_{j}+\overrightarrow{1} \mu_{l} . \\
& g_{2} \mu_{2}=\langle 1110 \mid 11\rangle^{2}\left(\mu_{l}+\mu_{i}+\mu_{j}\right) .
\end{aligned}
$$

We list the $g$ factor expressions of the tensor diquarks in SZ's model below:

$$
\begin{aligned}
& g_{u d}=\frac{m_{u d}}{2}\left[\frac{2}{m_{u}}-\frac{1}{m_{d}}+\frac{1}{m_{u}+m_{d}}\left(\frac{2 m_{d}}{m_{u}}-\frac{m_{u}}{m_{d}}\right)\right], \\
& g_{u s}=\frac{m_{u s}}{2}\left[\frac{2}{m_{u}}-\frac{1}{m_{s}}+\frac{1}{m_{u}+m_{s}}\left(\frac{2 m_{s}}{m_{u}}-\frac{m_{u}}{m_{s}}\right)\right], \\
& g_{d s}=\frac{m_{d s}}{4}\left[\frac{1}{m_{d}}+\frac{1}{m_{s}}+\frac{1}{m_{d}+m_{s}}\left(\frac{m_{s}}{m_{d}}+\frac{m_{d}}{m_{s}}\right)\right]
\end{aligned}
$$




\begin{tabular}{ccc}
\hline$\left(Y, I, I_{3}\right)$ & Decuplet Magnetic Moments & Numerical results \\
\hline$(2,0,0)$ & $\frac{1}{9}\left(g_{u d} \frac{1}{m_{u d}}-\frac{1}{2 m_{s}}\right) e_{0}$ & 0.23 \\
$\left(1, \frac{1}{2}, \frac{1}{2}\right)$ & $\frac{1}{27}\left(g_{u d} \frac{2}{m_{u d}}+g_{u s} \frac{1}{m_{u s}}-\frac{1}{m_{s}}-\frac{1}{2 m_{d}}\right) e_{0}$ & 0.26 \\
$\left(1, \frac{1}{2},-\frac{1}{2}\right)$ & $\frac{1}{27}\left(g_{u d} \frac{2}{m_{u d}}-g_{d s} \frac{2}{m_{d s}}-\frac{1}{m_{s}}+\frac{1}{m_{u}}\right) e_{0}$ & 0.09 \\
$(0,1,1)$ & $\frac{1}{27}\left(g_{u s} \frac{2}{m_{u s}}+g_{u d} \frac{1}{m_{u d}}-\frac{1}{m_{d}}-\frac{1}{2 m_{s}}\right) e_{0}$ & 0.30 \\
$(0,1,0)$ & $\frac{1}{27}\left(-g_{d s} \frac{2}{m_{d s}}+g_{u s} \frac{1}{m_{u s}}+g_{u d} \frac{1}{m_{u d}}+\frac{1}{m_{u}}-\frac{1}{2 m_{d}}-\frac{1}{2 m_{s}}\right) e_{0}$ & 0.13 \\
$(0,1,-1)$ & $\frac{1}{27}\left(-g_{d s} \frac{4}{m_{d s}}+g_{u d} \frac{1}{m_{u d}}+\frac{2}{m_{u}}-\frac{1}{2 m_{s}}\right) e_{0}$ & -0.04 \\
$\left(-1, \frac{3}{2}, \frac{3}{2}\right)$ & $\frac{1}{9}\left(g_{u s} \frac{1}{m_{u s}}-\frac{1}{2 m_{d}}\right) e_{0}$ & 0.33 \\
$\left(-1, \frac{3}{2}, \frac{1}{2}\right)$ & $\frac{1}{27}\left(g_{u s} \frac{2}{m_{u s}}-g_{d s} \frac{2}{m_{g s}}-\frac{1}{m_{d}}+\frac{1}{m_{u}}\right) e_{0}$ & 0.16 \\
$\left(-1, \frac{3}{2},-\frac{1}{2}\right)$ & $\frac{1}{27}\left(-g_{d s} \frac{4}{m_{d s}}+g_{u s} \frac{1}{m_{u s}}+\frac{2}{m_{u}}-\frac{1}{2 m_{d}}\right) e_{0}$ & -0.01 \\
$\left(-1, \frac{3}{2},-\frac{3}{2}\right)$ & $\frac{1}{9}\left(-g_{d s} \frac{2}{m_{d s}}+\frac{1}{m_{u}}\right) e_{0}$ & -0.17 \\
\hline
\end{tabular}

TABLE VI: Expressions of the magnetic moments of the anti-decuplet pentaquarks and numerical results of them in unit of $\mu_{N}$ in Shuryak and Zahed's model [19].

\begin{tabular}{ccc}
\hline$\left(Y, I, I_{3}\right)$ & Octet Magnetic Moments & Numerical results \\
\hline$\left(1, \frac{1}{2}, \frac{1}{2}\right)$ & $\frac{1}{27}\left(g_{u d} \frac{5}{2 m_{u d}}+g_{u s} \frac{1}{2 m_{u s}}-\frac{1}{m_{d}}-\frac{1}{2 m_{s}}\right) e_{0}$ & 0.20 \\
$\left(1, \frac{1}{2},-\frac{1}{2}\right)$ & $\frac{1}{27}\left(g_{u d} \frac{5}{2 m_{u d}}-g_{d s} \frac{1}{m_{d s}}+\frac{2}{m_{u}}-\frac{1}{2 m_{s}}\right) e_{0}$ & 0.55 \\
$(0,1,1)$ & $\frac{1}{27}\left(g_{u s} \frac{5}{2 m_{u s}}+g_{u d} \frac{1}{2 m_{u d}}-\frac{1}{m_{s}}-\frac{1}{2 m_{d}}\right) e_{0}$ & 0.35 \\
$(0,1,0)$ & $\frac{1}{54}\left(g_{u d} \frac{1}{m_{u d}}-g_{d s} \frac{5}{m_{d s}}+g_{u s} \frac{5}{2 m_{u s}}+\frac{1}{m_{u}}-\frac{2}{m_{s}}-\frac{1}{2 m_{d}}\right) e_{0}$ & -0.07 \\
$(0,1,-1)$ & $\frac{1}{27}\left(g_{u d} \frac{1}{2 m_{u d}}-g_{d s} \frac{5}{m_{d s}}+\frac{1}{m_{u}}-\frac{1}{m_{s}}\right) e_{0}$ & -0.50 \\
$\left(-1, \frac{1}{2}, \frac{1}{2}\right)$ & $\frac{1}{27}\left(g_{u s} \frac{5}{2 m_{u s}}-g_{d s} \frac{1}{m_{d s}}+\frac{2}{m_{u}}-\frac{1}{2 m_{d}}\right) e_{0}$ & 0.68 \\
$\left(-1, \frac{1}{2},-\frac{1}{2}\right)$ & $\frac{1}{27}\left(g_{u s} \frac{1}{2 m_{u s}}-g_{d s} \frac{1}{m_{d s}}+\frac{1}{m_{u}}-\frac{1}{m_{d}}\right) e_{0}$ & -0.53 \\
$(0,0,0)$ & $\frac{1}{18}\left(g_{u d} \frac{1}{m_{u d}}-g_{d s} \frac{1}{m_{d s}}+g_{u s} \frac{1}{2 m_{u s}}+\frac{1}{m_{u}}-\frac{1}{2 m_{d}}\right) e_{0}$ & 0.33 \\
\hline
\end{tabular}

TABLE VII: Expressions of the magnetic moments of the octet pentaquarks and their numerical results in unit of $\mu_{N}$ in Shuryak and Zahed's model [19].

$\Theta^{+}$'s magnetic moment in this case is:

$$
\begin{aligned}
\mu_{\Theta^{+}} & =\left\langle 2 \mu_{\bar{q}} \frac{\overrightarrow{1}}{2}+g_{2} \mu_{2} \overrightarrow{1}\right\rangle \\
& =\left(\left\langle 10 \frac{1}{2} \frac{1}{2} \mid \frac{1}{2} \frac{1}{2}\right\rangle^{2}-\left\langle 11 \frac{1}{2}-\frac{1}{2} \mid \frac{1}{2} \frac{1}{2}\right\rangle^{2}\right) \mu_{\bar{q}}+\left\langle 11 \frac{1}{2}-\frac{1}{2} \mid \frac{1}{2} \frac{1}{2}\right\rangle^{2} g_{2} \mu_{2}
\end{aligned}
$$

We present the expressions and numerical results in Table $\nabla \mathbf{T}$ and $\nabla \mathbf{I I}$ For quark masses, we use $m_{u}=m_{d}=360$ $\mathrm{MeV}, m_{s}=500 \mathrm{MeV}$. For the tensor diquark masses, we follow Ref. [19] and use $m_{u d}^{T}=570 \mathrm{MeV}, m_{u s}^{T}=m_{d s}^{T}=720$ $\mathrm{MeV}$. The scalar diquark mass is irrelevant in our calculation because there is no orbital excitation among the three constituents and the magnetic moment of the scalar diquark is zero. The pentaquark $\Theta^{+}$'s and $\Xi_{5}^{--}$'s magnetic moments are about $\mu_{\Theta^{+}}=0.37 \frac{e_{0}}{2 M_{\Theta^{+}}}$and $\mu_{\Xi_{5}^{--}}=-0.35 \frac{e_{0}}{2 M_{\Xi_{5}^{--}}}$respectively.

\section{PENTAQUARK AS A BOUND STATE OF A DIQUARK AND TRIQUARK}

An even simpler picture came from Karliner and Lipkin's diquark triquark model [18]. In their model the two color non-singlet clusters are kept apart by the P-wave angular momentum barrier. Hence the color magnetic interaction occurs within two clusters only. Between two clusters the color electric forces bind them into a color singlet. The angular momentum barrier prevents them from rearranging into the usual $K N$ system. The presence of $\mathrm{P}$-wave ensures the overall $\Theta^{+}$parity is positive.

The two quarks in the triquark are in the symmetric $\mathbf{6}_{\mathbf{c}}$ representation. They couple with the antiquark to form an $S U(3)_{c}$ triplet $\mathbf{3}_{c}$. The two quarks are in the anti-symmetric flavor $\overline{\mathbf{3}}_{\mathbf{f}}$ representation and the triquark is in the symmetric $\overline{\mathbf{6}}_{f}$. Thus the spin wave function of the two quarks is symmetric. The spin of the triquark is one half.

The direct product of the $\overline{\mathbf{3}}_{\mathbf{f}}$ of diquark and the $\overline{\mathbf{6}}_{\mathbf{f}}$ of triquark leads to $\overline{\mathbf{1 0}}_{\mathbf{f}}$ and $\mathbf{8}_{\mathbf{f}}$ pentaquarks. There is one orbital angular momentum $L=1$ between the diquark and the triquark. The resulting $J^{P}$ of the pentaquark is $\frac{1}{2}^{+}$. We list the flavor wave functions in Table VIII and IX] 


\begin{tabular}{ccc}
\hline$\left(Y, I, I_{3}\right)$ & Flavor wave functions & Magnetic moments \\
\hline$(2,0,0)$ & {$[u d]\{u d \bar{s}\}$} & 0.19 \\
$\left(1, \frac{1}{2}, \frac{1}{2}\right)$ & $\sqrt{\frac{1}{3}}([u d]\{u d \bar{d}\}+[u d]\{u s \bar{s}\}+[u s]\{u d \bar{s}\})$ & 0.17 \\
$\left(1, \frac{1}{2},-\frac{1}{2}\right)$ & $\sqrt{\frac{1}{3}}([u d]\{u d \bar{u}\}+[u d]\{d s \bar{s}\}+[d s]\{u d \bar{s}\})$ & -0.006 \\
$(0,1,1)$ & $\sqrt{\frac{1}{3}}([u s]\{u d \bar{d}\}+[u s]\{u s \bar{s}\}+[u d]\{u s \bar{d}\})$ & 0.15 \\
$(0,1,0)$ & $\sqrt{\frac{1}{6}}([u s]\{u d \bar{u}\}+[u s]\{d s \bar{s}\}+[d s]\{u d \bar{d}\}$ & -0.03 \\
& $+[d s]\{u s \bar{s}\}+[u d]\{u s \bar{u}\}+[u d]\{d s \bar{d}\})$ \\
$(0,1,-1)$ & $\sqrt{\frac{1}{3}}([d s]\{u d \bar{u}\}+[d s]\{d s \bar{s}\}+[u d]\{d s \bar{u}\})$ & -0.21 \\
$\left(-1, \frac{3}{2}, \frac{3}{2}\right)$ & {$[u s]\{u s \bar{d}\}$} & 0.13 \\
$\left(-1, \frac{3}{2}, \frac{1}{2}\right)$ & $\sqrt{\frac{1}{3}}([u s]\{u s \bar{u}\}+[u s]\{d s \bar{d}\}+[d s]\{u s \bar{d}\})$ & -0.054 \\
$\left(-1, \frac{3}{2},-\frac{1}{2}\right)$ & $\sqrt{\frac{1}{3}}([d s]\{d s \bar{d}\}+[d s]\{u s \bar{u}\}+[u s]\{d s \bar{u}\})$ & -0.24 \\
$\left(-1, \frac{3}{2},-\frac{3}{2}\right)$ & {$[d s]\{d s \bar{u}\}$} \\
\hline
\end{tabular}

TABLE VIII: Wave functions and numerical results of the magnetic moments of $\overline{\mathbf{1 0}}$ pentaquarks in unit of $\mu_{N}$ in Karliner and Lipkin's model $[18]$. $Y, I$ and $I_{3}$ are hypercharge, isospin and the third component of isospin respectively. $\left\{q_{1} q_{2} \overline{q_{3}}\right\} \equiv\left[q_{1} q_{2}\right] \overline{q_{3}}$ is the triquark's flavor wave function.

\begin{tabular}{ccc}
\hline$\left(Y, I, I_{3}\right)$ & Flavor wave functions & Magnetic moments \\
\hline$\left(1, \frac{1}{2}, \frac{1}{2}\right)$ & $\sqrt{\frac{1}{6}}([u d]\{u d \bar{d}\}+[u d]\{u s \bar{s}\})-\sqrt{\frac{2}{3}}[u s]\{u d \bar{s}\}$ & 0.16 \\
$\left(1, \frac{1}{2},-\frac{1}{2}\right)$ & $\sqrt{\frac{1}{6}}([u d]\{u d \bar{u}\}+[u d]\{d s \bar{s}\})-\sqrt{\frac{2}{3}}[d s]\{u d \bar{s}\}$ & -0.14 \\
$(0,1,1)$ & $\sqrt{\frac{1}{6}}([u s]\{u d \bar{d}\}+[u s]\{u s \bar{s}\})-\sqrt{\frac{2}{3}}[u d]\{u s \bar{d}\}$ & 0.16 \\
$(0,1,0)$ & $\sqrt{\frac{1}{12}}([u s]\{u d \bar{u}\}+[u s]\{d s \bar{s}\}+[d s]\{u d \bar{d}\}$ & 0.04 \\
& $+[d s]\{u s \bar{s}\})-\sqrt{\frac{1}{3}}([u d]\{u s \bar{u}\}+[u d]\{d s \bar{d}\})$ \\
$(0,1,-1)$ & $\sqrt{\frac{1}{6}}([d s]\{u d \bar{u}\}+[d s]\{d s \bar{s}\})-\sqrt{\frac{2}{3}}[u d]\{d s \bar{u}\}$ & -0.07 \\
$\left(-1, \frac{1}{2}, \frac{1}{2}\right)$ & $\sqrt{\frac{1}{6}}([u s]\{u s \bar{u}\}+[u s]\{d s \bar{d}\})-\sqrt{\frac{2}{3}}[d s]\{u s \bar{d}\}$ & -0.17 \\
$\left(-1, \frac{1}{2},-\frac{1}{2}\right)$ & $\sqrt{\frac{1}{6}}([d s]\{d s \bar{d}\}+[d s]\{u s \bar{u}\})-\sqrt{\frac{2}{3}}[u s]\{d s \bar{u}\}$ & -0.12 \\
$(0,0,0)$ & $\frac{1}{2}([u s]\{u d \bar{u}\}+[u s]\{d s \bar{s}\}-[d s]\{u d \bar{d}\}-[d s]\{u s \bar{s}\})$ & -0.10 \\
\hline
\end{tabular}

TABLE IX: Wave functions and numerical results of the magnetic moments of $\boldsymbol{8}_{\mathbf{f}}$ pentaquarks in unit of $\mu_{N}$ in Karliner and Lipkin's model [18]. $Y, I$ and $I_{3}$ are hypercharge, isospin and the third component of isospin respectively.

The intrinsic magnetic moment of the triquark is defined as

$$
g_{t r i} \mu_{t r i} \frac{\overrightarrow{1}}{2}=2 \mu_{q_{1}} \frac{\overrightarrow{1}}{2}+2 \mu_{q_{2}} \frac{\overrightarrow{1}}{2}+2 \mu_{\bar{q}} \frac{\overrightarrow{1}}{2}
$$

From the spin structure of the triquark, we get

$$
\frac{1}{2} g_{t r i} \mu_{t r i}=\left\langle 11 \frac{1}{2}-\frac{1}{2} \mid \frac{1}{2} \frac{1}{2}\right\rangle^{2}\left(\mu_{q_{1}}+\mu_{q_{2}}\right)+\left(\left\langle 10 \frac{1}{2} \frac{1}{2} \mid \frac{1}{2} \frac{1}{2}\right\rangle^{2}-\left\langle 11 \frac{1}{2}-\frac{1}{2} \mid \frac{1}{2} \frac{1}{2}\right\rangle^{2}\right) \mu_{\bar{q}}
$$

For the orbital part we have

$$
\begin{aligned}
& \mu_{l} \overrightarrow{1}=\mu_{t r i} \overrightarrow{l_{t r i}}+\mu_{d i} \overrightarrow{l_{d i}} \\
& \mu_{l}=\frac{m_{t r i} \mu_{d i}+m_{d i} \mu_{t r i}}{m_{t r i}+m_{d i}}
\end{aligned}
$$

where $m_{d i}$ is the mass of the diquark, $m_{t r i}$ is the mass of the triquark. 
Now the magnetic moment of the pentaquark is

$$
\begin{gathered}
\vec{\mu}=\mu_{l} \overrightarrow{1}+g_{t r i} \mu_{t r i} \frac{\overrightarrow{1}}{2} . \\
\mu=\left\langle 11 \frac{1}{2}-\frac{1}{2} \mid \frac{1}{2} \frac{1}{2}\right\rangle^{2} \mu_{l}+\left(\left\langle 10 \frac{1}{2} \frac{1}{2} \mid \frac{1}{2} \frac{1}{2}\right\rangle^{2}-\left\langle 11 \frac{1}{2}-\frac{1}{2} \mid \frac{1}{2} \frac{1}{2}\right\rangle^{2}\right) \frac{1}{2} g_{t r i} \mu_{t r i}
\end{gathered}
$$

The results are listed in Table VIII and IX] We use $m_{u}=m_{d}=360 \mathrm{MeV}, m_{s}=500 \mathrm{MeV}$. The mass of each diquark (triquark) is the sum of its constituent mass as in Ref. [18].

\section{MIT BAG MODEL}

Strottman discussed pentaquarks in the MIT bag model many years ago [29]. All five quarks in a low-lying pentaquark are in the same single particle orbital ground state with angular momentum $l=0$. Thus they have negative parity. One can classify the pentaquark states according to their spin, flavor and color symmetry $S U(6) \otimes S U_{f}(3) \supset$ $S U_{s}(2) \otimes S U(3)_{c} \otimes S U(3)_{f}$ as $[29]$

$$
\left|q^{4}[f](\lambda \mu)_{f}(10)_{c} \mathcal{S}_{q} I_{q} Y_{q} \times \bar{q}\left[1^{5}\right](01)_{f}(01)_{c} \frac{1}{2} I_{\bar{q}} Y_{\bar{q}} ;[f]_{t}(00)_{c} S I Y\right\rangle
$$

The pentaquark state is constructed from a four quark cluster $q^{4}$ and an anti-quark $\bar{q}$. Both the four quark cluster and the single anti-quark are specified by their $S U(6)$ representation $[f], S U_{f}(3)$ representation $(\lambda \mu)_{f}, S U(3)_{c}$ representation $(\lambda \mu)_{c}$, and quantum numbers spin $S_{i}$, isospin $I_{i}$ and hypercharge $Y_{i}$ respectively. The $(00)_{c}$ color singlet pentaquark state is further specified by its $S U(6)$ representation $[f]_{t}$, and quantum numbers spin $\mathcal{S}$, iso-spin $I$ and hypercharge $Y$. Since the anti-quark is in color $(01)_{c}$ representation, the four quark cluster must be in $(10)_{c}$ to form a color singlet pentaquark state. From the Pauli principle, the total wave function of the four quark cluster must be anti-symmetric which requires that its $S U_{f}(3)$ representation $(\lambda \mu)_{f}$ is conjugated to the $S U(6)$ representation $[f]$. The calculation [29] showed that all the $\frac{1}{2}^{-}$pentaquarks with mass less than $1900 \mathrm{MeV}$ belong to the single $S U(6)$ representation [21].

For the $\Theta^{+}$particle, its quantum numbers are $\left(J, Y, I, I_{3}\right)=\left(\frac{1}{2}, 2,0,0\right)$. The lowest energy state was predicted around $1.70 \mathrm{GeV}$ and has the four quark cluster in $S U(6)$ representation [22] (the conjugated $S U_{f}(3)$ representation of the four quark cluster is $\left.(02)_{f}\right)$. With the Clebsch-Gordan coefficients we get [29],

$$
\begin{aligned}
& \left|q^{4}[22](02)_{f} I_{q} Y_{q} \times \bar{q}\left[1^{5}\right](01)_{f} I_{\bar{q}} Y_{\bar{q}} ;[21](00)_{c} \frac{1}{2} I Y\right\rangle \\
= & \left|q^{4}[22](02)_{f}(10)_{c} 1 I_{q} Y_{q} \times \bar{q}\left[1^{5}\right](01)_{f}(01)_{c} \frac{1}{2} I_{\bar{q}} Y_{\bar{q}} ;(00)_{c} \frac{1}{2} I Y\right\rangle .
\end{aligned}
$$

Hence the spin of the four quark cluster must be $S=1$. As the iso-spin of the four quark cluster in $\Theta^{+}$is $T=0$, its mangetic moment can be obtain from the average mangetic moment of u- and d- quarks $\bar{\mu}=\frac{1}{2}\left(\mu_{u}+\mu_{d}\right)$

$$
\mu_{q^{4}}=2 \bar{\mu}=\mu_{u}+\mu_{d}
$$

where 2 is the $g$-factor of quarks. The pentaquark magnetic moment is

$$
\begin{aligned}
\mu_{\Theta^{+}} & =\frac{2}{3} \mu_{q^{4}}-\frac{1}{3} \mu_{\bar{q}} \\
& =\frac{2 e_{0}}{9 m_{u}}-\frac{e_{0}}{9 m_{d}}-\frac{e_{0}}{18 m_{s}}=\frac{e_{0}}{9 m_{u}}-\frac{e_{0}}{18 m_{s}}
\end{aligned}
$$

For the $\Xi_{5}^{--}$and $\Xi_{5}^{+}$particle, their quantum numbers are $\left(J, Y, I, I_{3}\right)=\left(\frac{1}{2},-1, \frac{3}{2},-\frac{3}{2}\right)$ and $\left(J, Y, I, I_{3}\right)=$ $\left(\frac{1}{2},-1, \frac{3}{2}, \frac{3}{2}\right)$ respectively [29]. The lowest energy state was predicted around $1.90 \mathrm{GeV}$ with the four quark cluster still in $S U(6)$ representation [22]. Similarly, we obtain

$$
\begin{aligned}
\mu_{\Xi_{5}^{--}} & =\frac{2}{3}\left(\mu_{d}+\mu_{s}\right)-\frac{1}{3} \mu_{\bar{u}} \\
& =-\frac{e_{0}}{9 m_{d}}-\frac{e_{0}}{9 m_{s}}+\frac{e_{0}}{9 m_{u}}=-\frac{e_{0}}{9 m_{s}},
\end{aligned}
$$


and

$$
\begin{aligned}
\mu_{\Xi_{5}^{+}} & =\frac{2}{3}\left(\mu_{u}+\mu_{s}\right)-\frac{1}{3} \mu_{\bar{d}} \\
& =\frac{2 e_{0}}{9 m_{u}}-\frac{e_{0}}{9 m_{s}}-\frac{e_{0}}{18 m_{d}}=\frac{e_{0}}{6 m_{u}}-\frac{e_{0}}{9 m_{s}} .
\end{aligned}
$$

If the four quark cluster in a pentaquark favors the $S U(6)$ representation [22], the flavor of the four quark cluster will be the conjugated $S U_{f}(3)$ representation $(02)_{f}$. The total flavor symmetry of the pentaquark states will be

$$
(02) \times(01)=(11)+(03),
$$

including an $\mathbf{8}_{f}$ and a $\overline{\mathbf{1 0}}_{f}$ (clearly, the above exotic states belong to the $\overline{\mathbf{1 0}}_{f}$ representation). The magnetic moments of all these pentaquark states are

$$
\mu=\frac{2}{3} \mu_{q^{4}}-\frac{1}{3} \mu_{\bar{q}}
$$

with

$$
\mu_{q^{4}}=2 \bar{\mu}=\frac{1}{2} \sum \mu_{q_{i}}
$$

\section{DISCUSSIONS}

Several theoretical papers have already discussed the $\Theta^{+}$magnetic moments. In Ref. 31 Kim and Praszalowicz derived relations for the anti-decuplet magnetic moments within the framework of chiral soliton model in the chiral limit. The $\Theta^{+}$pentaquark magnetic moment is estimated to be $(0.2 \sim 0.3) \mu_{N}$ [31].

In Ref. [32], Nam, Hosaka and Kim first estimated the anomalous magnetic moment $\kappa_{\Theta^{+}}$of the $\Theta^{+}$pentaquark when they discussed the photoproduction of $\Theta^{+}$from the neutron. For example, they have estimated $\kappa_{\Theta^{+}}=-0.7$ and $\kappa_{\Theta^{+}}=-0.2$ for $J^{P}=\frac{1}{2}^{+}$and $J^{P}=\frac{1}{2}^{-}$respectively using Jaffe and Wilczek's diquark picture. Also, they have considered the possibility of a $\mathrm{K} \mathrm{N}$ bound system, with $\kappa\left(K N, J^{P}=\frac{1}{2}^{+}\right)=-0.4$ and $\kappa\left(K N, J^{P}=\frac{1}{2}^{-}\right)=-0.5$. These numbers are all in unit of $\Theta^{+}$magneton $\frac{e_{0}}{2 m_{\Theta^{+}}}$.

A quark model calculation of the $\Theta^{+}$magnetic moment using Jaffe and Wilczek's model was also performed in Ref. [30], where Zhao arrived at

$$
\mu_{\Theta^{+}}=0.13 \frac{e_{0}}{2 m_{\Theta^{+}}}
$$

for the positive parity $\Theta^{+}$pentaquark. He also discussed the possibility of $\Theta^{+}$parity being negative. In this case he estimated its magnetic moment as the sum of $(u \bar{s})$ and $(u d d)$ clusters. Numerically

$$
\mu_{\Theta^{+}}=\frac{e_{0}}{6 m_{s}} .
$$

We have estimated the magnetic moment of the $\Theta^{+}$pentaquark state in the framework of light cone QCD sum rules using the photon distribution amplitude. We have obtained a quite small value: $\left|\mu_{\Theta^{+}}\right|=(0.12 \pm 0.06) \mu_{N}$ [28].

In Table $\$ we have collected all available predictions of the magnetic moments for $\Theta^{+}, \Xi_{5}^{--}$and $\Xi_{5}^{+}$pentaquarks. There may exist strong mixing between the other $\overline{\mathbf{1 0}}_{f}$ members and the corresponding octet pentaquarks. Experimentally it will be very difficult to distinguish them. So we focus on the $\Theta^{+}, \Xi_{5}^{--}$and $\Xi_{5}^{+}$pentaquarks which lie on the corners of the $\overline{\mathbf{1 0}}_{f}$ triangle and admit no mixing. It's interesting to note that different models tend to yield relatively close numerical results for the $\Theta^{+}$magnetic moment. But their predictions for $\Xi_{5}^{--}$and $\Xi_{5}^{+}$vary significantly, which in turn will affect the photo-production or electro-production total and differential cross sections. With the accumulation of events, pentaquark magnetic moments may be extracted from the comparison of theoretical and experimental cross sections eventually in the near future, which may help us distinguish different models and deepen our understanding of the underlying dynamics governing their formation.

This project was supported by the National Natural Science Foundation of China under Grant 10375003, Ministry of Education of China, FANEDD and SRF for ROCS, SEM.

[1] T. Nakano et al., Phys. Rev. Lett. 91, 012002 (2003). 


\begin{tabular}{c|c|c|c|c}
\hline & $\Theta^{+}$ & $\Xi_{5}^{--}$ & $\Xi_{5}^{+}$ \\
\hline Ref. [31] & $0.2 \sim 0.3$ & -0.4 & 0.2 \\
\hline Ref. [32] & $0.2 \sim 0.5$ & - & - \\
\hline Ref. [30] & $0.08 \sim 0.6$ & - & - \\
\hline Ref. [28] & $(0.12 \pm 0.06)$ & - & - \\
\hline \multirow{3}{*}{$\begin{array}{c}\text { Present } \\
\text { Work }\end{array}$} & Jaffe and Wilczek's model & 0.08 & 0.12 & -0.06 \\
\cline { 2 - 5 } & Shuryak and Zahed's model & 0.23 & -0.17 & 0.33 \\
\cline { 2 - 5 } & Karliner and Lipkin's model & 0.19 & -0.43 & 0.13 \\
\cline { 2 - 5 } & Strottman's model & 0.37 & -0.42 & 0.45 \\
\hline
\end{tabular}

TABLE X: Comparison of magnetic moments of $\Theta^{+}, \Xi_{5}^{--}$and $\Xi_{5}^{+}$from other group's calculation with the present work using different pentaquark models in literature. The numbers are in unit $\mu_{N}$.

[2] V. V. Barmin et al., hep-ex/0304040

[3] S. Stepanyan et al., hep-ex/0307018

[4] J. Barth et al., hep-ph/0307083

[5] A. E. Aratayn, A. G. Dololenko and M. A. Kubantsev, hep-ex/0309042

[6] NA49 Collaboration, hep-ex/0310014

[7] V. Kubarovsky et al., hep-ex/0311046

[8] T. D. Cohen, Phys. Lett. B 427, 348 (1998).

[9] Particle Dada Group, Phys. Rev. D 66, 010001 (2002).

[10] D. Diakonov, V. Petrov, and M. Ployakov, Z. Phys. A 359, 305 (1997).

[11] S. Capstick, P. R. Page, and W. Roberts, hep-ph/0307019

[12] T. D. Cohen, R. F. Lebed, hep-ph/0309150 T. D. Cohen, hep-ph/0309111

[13] N. Itzhaki et al., hep-ph/0309305

[14] Shi-Lin Zhu, hep-ph/0307345 Phys. Rev. Lett. 91, 232002 (2003).

[15] R.D. Matheus et al., hep-ph/0309001 J. Sugiyama, T. Doi, M. Oka, hep-ph/0309271

[16] F.Csikor, Z. Fodor, S.D. Katz, T.G. Kovacs, hep-lat/0309090 S. Sasaki, hep-lat/0310014

[17] R. Jaffe and F. Wilczek, Phys. Rev. Lett. 91, 232003 (2003).

[18] M. Karliner and H. J. Lipkin, hep-ph/0307243

[19] E. Shuryak and I. Zahed, hep-ph/0310270

[20] J. J. Dudek and F. E. Close, hep-ph/0311258

[21] F.Huang, Z.Y.Zhang, Y.W.Yu, B.S.Zou, hep-ph/0310040

[22] C. E. Carlson et al., hep-ph/0307396

[23] B. Wu and B.-Q. Ma, hep-ph/0311331

[24] S. Nussinov, hep-ph/0307357

[25] R. A. Arndt, I. I. Strakovsky, R. L. Workman, Phys. Rev. C 68, 042201 (R) (2003).

[26] J. Haidenbauer and G. Krein, hep-ph/0309243

[27] R. N. Cahn and G. H. Trilling, hep-h/0311245

[28] Peng-Zhi Huang, Wei-Zhen Deng, Xiao-Lin Chen, Shi-Lin Zhu, hep-ph/0311108

[29] D. Strottman, Phys. Rev. D 20, 748 (1979).

[30] Q. Zhao, hep-ph/0310350

[31] H.-C. Kim and M. Praszalowicz, hep-ph/0308242

[32] S. I. Nam, A. Hosaka, H.-Ch. Kim, hep-ph/0308313

[33] H. Gao and B.-Q. Ma, hep-ph/0305294 Mod. Phys. Lett. A 14, 2313 (1999);M. V. Ployakov and A. Rathke, hep-ph/0303138 F. Stancu and D. O. Riska, hep-ph/0307010 B. G. Wybourne, hep-ph/0307170 A. Hosaka, hep-ph/0307232 T. Hyodo, A. Hosaka, E. Oset, nucl-th/0307105 M. Karliner and H. J. Lipkin, hep-ph/0307343

[34] P.V. Pobylitsa, hep-ph/0310221 D. Diakonov, V. Petrov, hep-ph/0310212 J. Letessier, G. Torrieri, S. Steinke, J. Rafelski, hep-ph/0310188 I.M.Narodetskii et al., hep-ph/0310118 N. Auerbach, V. Zelevinsky, nucl-th/0310029 D.E. Kahana, S.H. Kahana, hep-ph/0310026 D. Diakonov, V. Petrov, hep-ph/0309203 L. Ya. Glozman, hep-ph/0309092 hep-ph/0308232 M. Praszalowicz, hep-ph/0308114 X. Chen, Y. Mao, B.-Q. Ma, hep-ph/0307381 S. Nussinov, hep-ph/0307357 J. Randrup, nucl-th/0307042 M.V. Polyakov, A. Rathke, hep-ph/0303138 H. Walliser, V.B. Kopeliovich, hep-ph/0304058

[35] R. Bijker, M.M. Giannini, E. Santopinto, hep-ph/0310281 Y. Oh, H. Kim, S. H. Lee, hep-ph/0310117 hep-ph/0310019 hep-ph/0311054 C. E. Carlson et al., hep-ph/0310038 B. Jennings, K. Maltman, hep-ph/0308286 K. Cheung, hep-ph/0308176 P.Bicudo, G. M. Marques, hep-ph/0308073 L. W. Chen, V. Greco, C. M. Ko, S. H. Lee, W. Liu, nucl-th/0308006 W. Liu, C. M. Ko, V. Kubarovsky, nucl-th/0310087 W. Liu, C. M. Ko, nucl-th/0309023 nucl-th/0308034 D. Borisyuk, M. Faber, A. Kobushkin, hep-ph/0307370 Felipe J. Llanes-Estrada, E. Oset, V. Mateu, nucl-th/0311020 D. Akers, hep-ph/0311031 K. Nakayama and K. Tsushima, hep-ph/0311112 A. R. Dzierba et al., hep-ph/0311125 R. A. Arndt, I. I. Strakovsky and R. L. Workman, nucl-th/0311030 D. P. Roy, hep-ph/0311207 M. Praszalowicz, hep-ph/0311230 F. E. Close, hep-ph/0311087 T. Kishimoto and T. Sato, hep-ex/0312003 